\title{
Paternidade na adolescência: uma breve revisão da literatura internacional $^{1}$
}

\author{
Daniela Centenaro Levandowski \\ Universidade Federal do Rio Grande do Sul \\ Universidade do Oeste de Santa Catarina - Chapecó
}

\begin{abstract}
Resumo
Este trabalho teve como objetivo revisar os artigos publicados no período de 1990 a 1999 sobre o tema da paternidade adolescente em âmbito internacional. A partir de uma consulta à base de dados Psyclit, utilizando termos descritores relacionados ao assunto, verificou-se a incidência de estudos sobre a paternidade e a maternidade em geral, e a paternidade e maternidade adolescentes. Após isso, foi feita a identificação dos resumos dos artigos que se referiam diretamente a pais adolescentes, encontrando-se apenas 68 resumos. Estes foram classificados de acordo com o tema investigado e alguns aspectos metodológicos. Constata-se a carência de estudos sobre o tema em questão, quando comparados aos estudos sobre o tema da paternidade em geral e da maternidade adolescente. Além disso, a necessidade de se investigar de forma mais aprofundada expectativas e sentimentos destes adolescentes, sua relação conjugal, com a família de origem e com o bebê. Instrumentos como testes, observação e entrevistas poderiam ser utilizados de forma mais sistemática.
\end{abstract}

Palavras-chave: Paternidade adolescente, Revisão, Literatura internacional.

\begin{abstract}
Adolescent fatherhood: Reviewing the international literature. This paper aimed to review the international literature on adolescent fatherhood published from 1990 to 1999 . The Psyclit data base was accessed using specific key words related to the theme. The incidence of studies on fatherhood, motherhood and adolescent fatherhood and motherhood was assessed. Thus, an identification of specific articles on adolescent fathers or fatherhood was carried out. Only 68 articles were found. These articles were classified according to their theme and some methodological aspects. A low incidence of articles on adolescent fatherhood was noted, compared to articles on fatherhood in general and adolescent motherhood. It is still necessary to investigate the expectations and feelings of adolescent fathers, their marital and family relationships and their involvement with the baby. Instruments such as tests, interviews and observation could be employed more systematically.
\end{abstract}

Key words: Adolescent fatherhood, Review, International literature.

A partir de consultas exploratórias à base de dados Psyclit, constatou-se um pequeno número de resumos de artigos sobre o tema da paternidade adolescente. Esta carência teórica e prática parece ser contraditória, em vista do grande número de reportagens veiculadas pela imprensa em relação ao aumento bombástico do número de gestações na adolescência em âmbito internacional.

Questionamentos em relação a esta ausência foram levantados: por que praticamente não se estuda o pai adolescente? Que impacto a paternidade teria nas diversas esferas de sua vida? O que já havia sido escrito sobre o tema no âmbito internacional e nacional? Estes questionamentos motivaram a realização deste trabalho de revisão da literatura internacional. Este enfoque baseou-se na existência de poucos estudos realizados sobre o pai adolescente no meio acadêmico nacional. Assim, nesse momento foi necessário recorrer à produção internacional, principalmente norte-americana, para que a elaboração deste trabalho se concretizasse.

Sabe-se que pesquisas de revisão de literatura têm um grande valor científico, por fornecerem, de forma resumida, um panorama abrangente sobre um determinado tema, ressaltando tanto os temas de pesquisa mais investigados pelos pesquisadores em determinada época, como também os temas que têm recebido pouca atenção dos mesmos (Piccinini \& Lopes, 1994). A partir dessa premissa, pode-se também realizar uma investigação mais detalhada sobre a elabora- 
ção teórica e a metodologia empregada, o que fornece uma idéia do nível de desenvolvimento da pesquisa e as suas possíveis contribuições ao meio acadêmico (Piccinini \&Lopes, 1994).

Assim, o objetivo geral deste trabalho foi realizar uma breve revisão da literatura internacional sobre o tema da paternidade adolescente. Buscou-se verificar a incidência de estudos sobre o tema da paternidade e da maternidade; bem como a incidência de estudos sobre a paternidade e a maternidade adolescentes, no período de 1974 - 1999. Posteriormente, examinou-se os artigos do período 1990-1999 que se referiam especificamente à paternidade adolescente, efetuando-se uma categorização quanto ao tema pesquisado e alguns aspectos metodológicos. Não se teve o objetivo de fazer uma revisão exaustiva do que foi produzido neste período, mas sim de examinar um número representativo de resumos de artigos publicados, no caso, aqueles cujo acesso foi possibilitado pela base de dadosPsyclit. Devido à confiança dos pesquisadores nessa base de dados e seus critérios rigorosos de indexação de periódicos, acredita-se ter englobado artigos mais relevantes sobre o tema.

\section{Método}

Primeiramente, objetivou-se verificar a incidência de artigos publicados desde 1974 a 1999, em revistas indexadas na base de dados Psyclit, sobre o tema da paternidade e da maternidade, e da paternidade e maternidade adolescentes especificamente. Para tanto, foram utilizados como descritores os termos: father and fatherhood, mother and motherhood, father or fatherhood, mother or motherhood, adolescent fatherhood, adolescent motherhood, adolescent father, adolescent mother, teenage father, teenage mother, parenthood, adolescent parenthood, teenage parenthood, teenage parent, adolescent parent. Estes termos, embora indicassem algumas vezes artigos repetidos, em outras vezes revelavam artigos não contidos em outros descritores. Assim, foi possível obter garantia a respeito da maior abrangência das consultas realizadas, que foram divididas em três períodos de tempo (1974 a 1990; 1991 a 1995 e 1996 a 1999).

Em seguida, foram examinados os resumos de artigos publicados especificamente sobre a paternidade adolescente, no período de 1990 a 1999, quanto ao tema estudado e alguns aspectos metodológicos. Para esta análise mais detalhada foram considerados apenas os resumos de artigos incluídos nos descritores adolescent father, teenage father, adolescent fatherhood, teenage fatherhood, adolescent parent e teenage parent. Foram excluídos resumos de capítulos ou livros que constavam dentro destes termos-chave. Tomou-se o cuidado de conferir todos os títulos e autores, evitando-se considerar o mesmo resumo mais de uma vez.
Para a classificação quanto ao tema estudado, foi realizada uma leitura inicial, que deu origem a categorias temáticas, nas quais os resumos foram alocados em uma leitura posterior. O mesmo ocorreu na análise dos aspectos metodológicos. Após isso, foi feita uma análise dos temas de todos os resumos classificados em cada categoria, a fim de se obter um panorama detalhado da produção científica internacional sobre a paternidade adolescente.

\section{Resultados e discussão}

Inicialmente, estão expostos os resultados encontrados na consulta inicial, realizada com os descritores acima citados, sobre a incidência de estudos sobre o tema da paternidade e da maternidade em geral (Figura 1), e da paternidade e maternidade adolescentes em particular (Figura 2). Na coluna incidência, o leitor pode encontrar o número de artigos realizados sobre o tema em questão.

Primeiramente, deve ser ressaltado o fato de que esses números servem apenas como uma ilustração da produção científica sobre o tema, uma vez que os artigos se repetem dentro de diferentes descritores. Contudo, não deixam de ilustrar, de forma bastante representativa, o "estado da arte" no que diz respeito a pesquisas envolvendo pais e mães, adolescentes e adultos. Também estão incluídos os capítulos e livros publicados sobre o tema e/ou relacionados a ele, excluídos da análise posterior.

Analisando-se conjuntamente os dois gráficos, é possível verificar uma diferença gritante no número de artigos e livros publicados sobre os dois temas, com uma maior incidência de pesquisas sobre o tema da maternidade. Conforme Elster e Lamb (1986), o pai parece ter sido esquecido, por muito tempo, como figura importante para o desenvolvimento psicológico da díade mãe-criança. De fato, ao longo dos anos não se percebe aumento do número de estudos sobre o tema da paternidade.

De modo mais específico, é marcante a baixa incidência de estudos referentes ao tema da paternidade na adolescência, indicados principalmente pelos termos adolescent father, teenage father, adolescent fatherhood e teenage fatherhood ( $\mathrm{n}=238)$. Juntos, os resumos contidos nestes descritores representam apenas $2,9 \%$ do total de artigos encontrados. Nesse sentido, é importante ressaltar que os descritores adolescent parenthood e adolescent parent (e respectivas combinações do termo teenage), embora pareçam fazer referência direta ao tema, englobam artigos que não tratam apenas de pais adolescentes, não sendo por isso considerados neste trabalho.

Pode-se notar que a situação é semelhante para o tema da maternidade adolescente. Os resumos contidos nos descritores adolescent mother, teenage mother, adolescent 


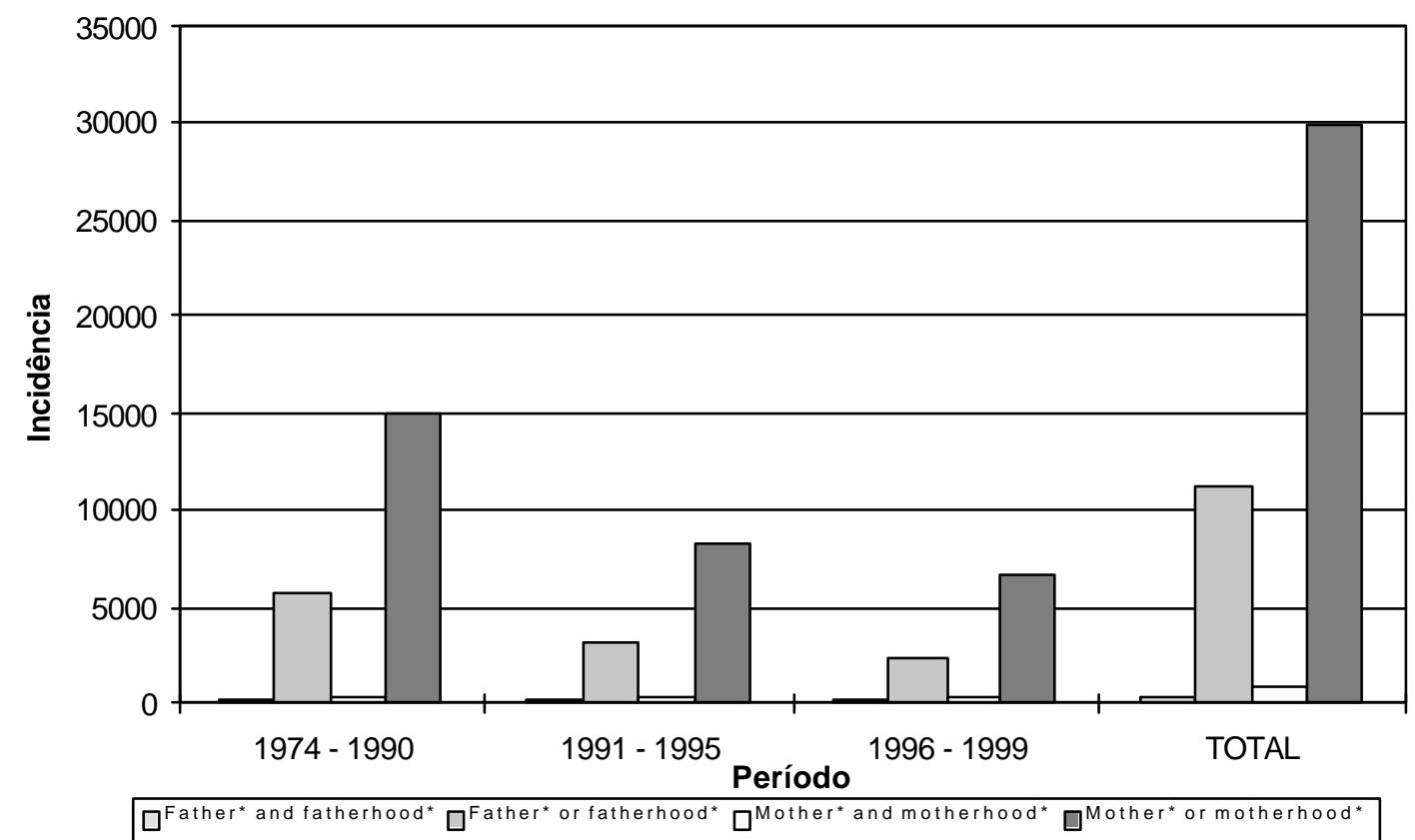

Figura 1. Incidência de estudos sobre paternidade e maternidade, de acordo com o período da consulta ao Psyclit

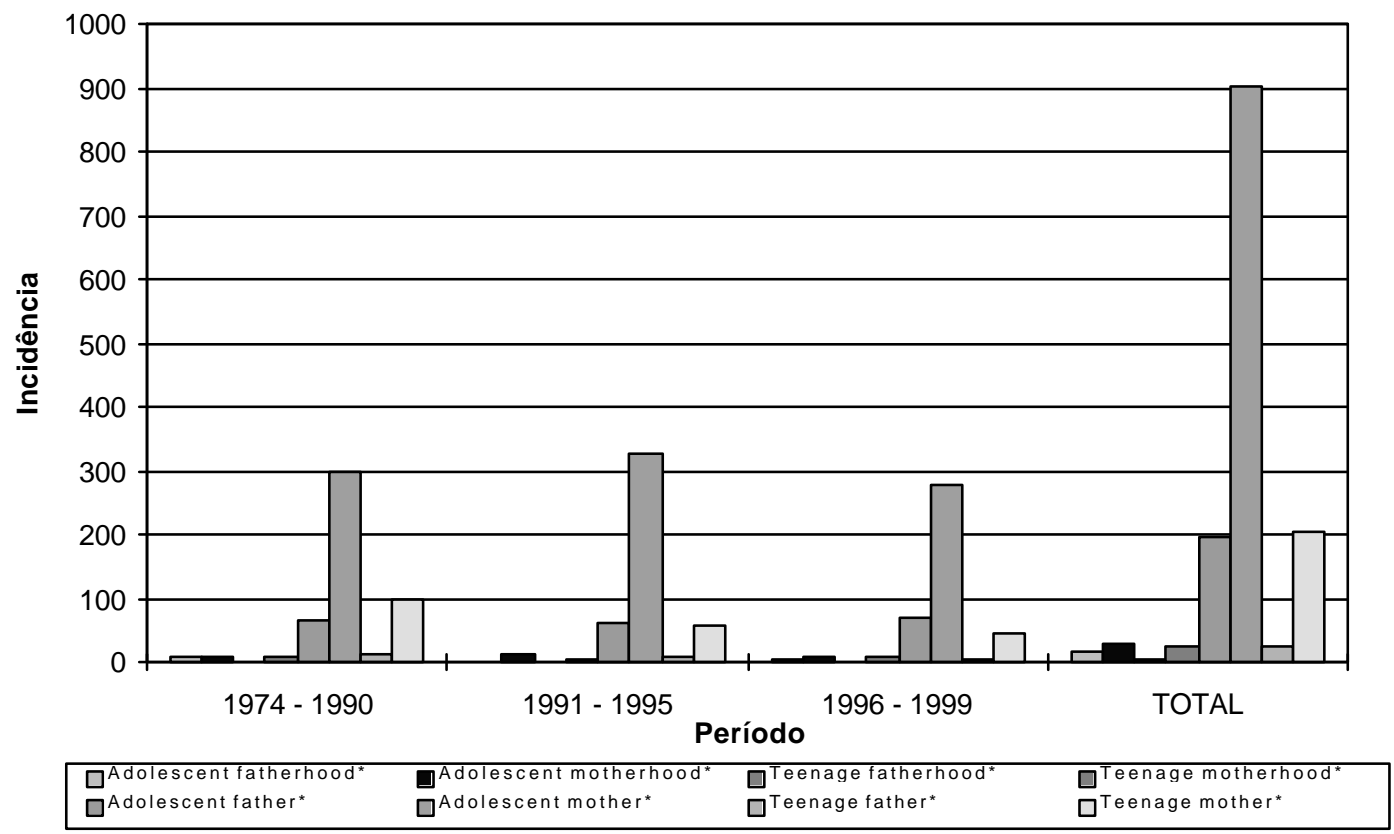

Figura 2. Incidência de estudos sobre paternidade adolescente e maternidade adolescente, de acordo com o período da consulta ao Psyclit 
motherhood e teenage motherhood $(\mathrm{n}=1158)$ correspondem a apenas 3,42\% do total de artigos encontrados sobre o tema da maternidade. Como no caso anterior, nota-se que há um número reduzido de estudos sobre o tema da maternidade adolescente em comparação aos artigos que versam sobre o tema da maternidade.

No entanto, fica evidente em todos os descritores relacionados ao tema da maternidade e paternidade adolescentes, a incidência superior de estudos sobre a maternidade adolescente. Esse achado confirma a tendência apontada pela literatura existente sobre paternidade adolescente, no que se refere ao foco das pesquisas sobre gestação na adolescência (Fonseca, 1998; Hendricks \& Montgomery, 1983). Em geral, estas têm levado em conta apenas a gestante/mãe adolescente, ignorando aspectos como preocupações de pais adolescentes, impacto da gestação e da paternidade, interação com o bebê, dentre outros.

O segundo passo foi a identificação de todos os artigos que se referiam especificamente à paternidade adolescente. Foram encontradas referências diretas em 68 resumos apenas (Anexo). Foi feita uma leitura inicial destes resumos, contidos nos descritores adolescent father, teenage father, adolescent fatherhood, teenage fatherhood, adolescent parent e teenage parent, a fim de se classificar tais artigos quanto ao tema estudado ou tipo de estudo. Foram identificadas as seguintes categorias:

- Revisão de literatura: nesta categoria foram incluídos todos os resumos que mencionavam a realização de uma revisão da literatura sobre o tema da paternidade adolescente, seja de forma geral ou específica (ex.: intervenções);
- Causas/Antecedentes/Fatores de risco: nessa categoria foram incluídos todos os resumos de artigos que investigassem possíveis causas, antecedentes ou fatores de risco para a ocorrência da paternidade adolescente (ex.: fatores econômicos, pessoais, sociais etc.);

- Consequiências/Resultados: nessa categoria foram incluídos todos os resumos de artigos que investigassem possíveis conseqüências ou resultados (positivos ou negativos) da paternidade adolescente para os próprios pais adolescentes, seus bebês e/ou suas parceiras (economicamente, pessoalmente, socialmente etc.);

- Aspectos familiares/conjugais: nessa categoria foram incluídos todos os resumos de artigos que investigassem especificamente aspectos da família de origem ou atual de pais adolescentes, bem como seu relacionamento com a mãe do bebê (antes ou depois da gestação);

- Experiências/Percepções/Vivências: nessa categoria foram incluídos todos os resumos de artigos que investigassem sentimentos, percepções, estresses etc., vividos tanto pelos futuros pais adolescentes como por aqueles adolescentes que já eram pais. Além disso, também englobavam resumos que investigavam a relação entre estes e seu bebê.

- Intervenção/Aconselhamento: nesta categoria foram incluídos todos os resumos em que estavam descritos resultados e/ou técnicas de intervenções e programas de aconselhamento, o papel dos profissionais envolvidos com intervenção/aconselhamento etc.;

- Outros: nessa categoria foram incluídos todos os resumos de artigos que não se enquadravam em nenhuma outra categoria. Foram incluídos resumos que tratavam de fa-

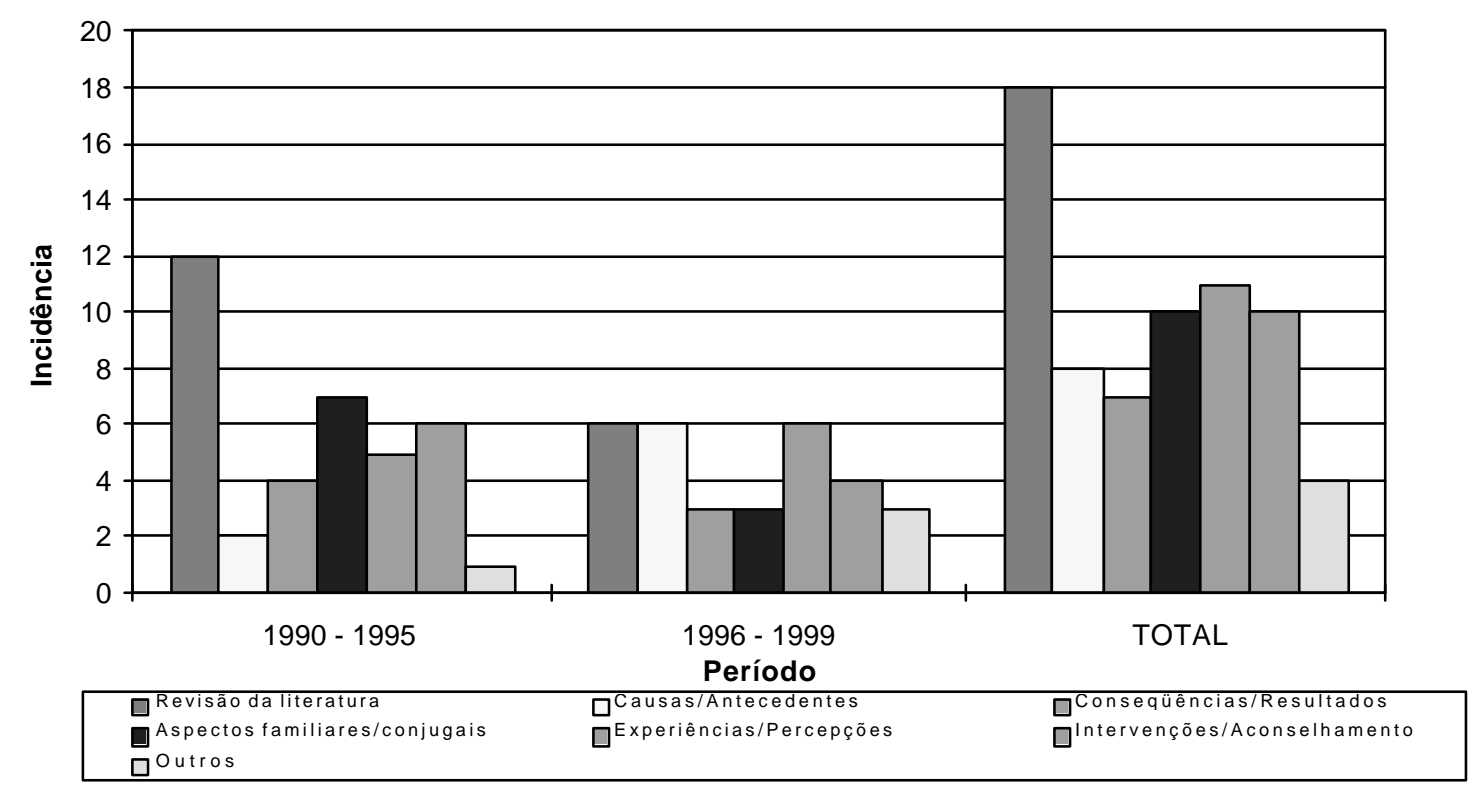

Figura 3. Incidência de estudos sobre paternidade adolescente, de acordo com o tema investigado e o período de consulta ao Psyclit 
tores apontados simultaneamente como possíveis antecedentes e conseqüências da paternidade adolescente.

Os resultados da análise estão expostos na Figura 3. Após isso, realizou-se uma análise dos resumos de cada uma das categorias citadas acima.

\section{Revisão de literatura}

Nesta categoria, a partir de uma análise dos resumos, observa-se que a grande maioria dos artigos revisa aspectos relacionados a intervenções e aconselhamento de pais adolescentes. São abordados temas como a necessidade de assistência (Girard, Coll \& Becco, 1991a; Kiselica, Stroud, Stroud \& Rotzien, 1992), o papel dos profissionais que aconselham estes jovens (Kiselica \& Pfaller, 1993), os serviços disponíveis (Kiselica \& Sturmer, 1993) e dados de atendimento dos mesmos (Kirby, 1991), bem como abordagens para intervenção (Joshi \& Battle, 1990; Kiselica \& Murphy, 1994; Marsiglio, 1995; Moroz \& Allen, 1991) e considerações políticas e históricas sobre o atendimento de pais adolescentes (Weatherley, 1991) e sobre a paternidade adolescente (Miller, 1992).

O restante dos artigos investiga temas diversos, relacionados ao pai adolescente, tais como o papel dos fatores psicológicos para a ocorrência da paternidade adolescente (Benoit, 1997), sua função de suporte para a mãe adolescente e o bebê (Roye \& Balk, 1996) e seu envolvimento com o bebê (Miller, 1997). Alguns tratam ainda da questão da transferência familiar do risco para a gravidez na adolescência (Serbin \& Stack, 1998), da paternidade de negros estadunidenses (Cochran, 1997) e da integração de pesquisas de abuso infantil e gravidez na adolescência (Becker \& Rickel, 1995; Stevens-Simon \& Nelligan, 1998).

De modo geral, os resumos revisam pesquisas, políticas sociais e/ou dados das agências prestadoras de serviço a pais adolescentes. É interessante notar que todos os resumos mencionam problemas, preocupações e estresses de pais adolescentes. Além disso, em sua maioria sugerem áreas para intervenções futuras com estes pais, demonstrando talvez uma ineficiência dos programas e serviços existentes até o presente momento. Isso evidenciaria a ausência de conhecimentos consistentes sobre o fenômeno da paternidade adolescente, uma vez que a falta destes pressupõe que as intervenções realizadas não estejam atingindo estes pais ou adolescentes que correm risco de se tornarem pais. Assim, novas pesquisas sobre o tema deveriam ser realizadas.

O grande número de artigos revisando intervenções poderia indicar que no meio acadêmico se está trilhando o caminho da paternidade adolescente talvez de forma contrária: antes de conhecer o fenômeno detalhadamente, se quer intervir de qualquer forma, a fim de conter o avanço do que já está sendo considerado uma epidemia, a gravidez na adolescência (Dimenstein, 1999; Soares, 1999).

\section{Causas/Antecedentes/Fatores de risco}

Nessa categoria, nota-se um número maior de artigos publicados a partir de 1996, o que parece mostrar a tomada de consciência dos profissionais envolvidos com a paternidade adolescente de que, para intervir de forma eficaz, seria necessário primeiramente conhecer este evento de forma consistente.

Dentre os resumos, são citados como fatores de risco específicos relacionados à paternidade adolescente: maus tratos precoces (Herrenkohl, Herrenkohl, Egolf \& Russo, 1998), uso de substâncias (Krohn, Lizotte \& Perez, 1997), distúrbios psiquiátricos (Kessler, Berglund, Foster, Saunders, Stang \& Walters, 1997), fracasso escolar (Dearden Hale \& Alvarez, 1992) e comportamento anti-social (Scaramella, Conger, Simons \& Whitbeck, 1998). Alguns artigos trazem o tema dos fatores de risco de forma geral, considerando-os simultaneamente (Dearden, Hale \& Woolley, 1995; Guagliardo, Huang \& D’Angelo, 1999; Thornberry, Smith \& Howard, 1997).

A grande maioria dos estudos tem caráter longitudinal (Dearden Hale \& Alvarez, 1992; Dearden, Hale \& Woolley, 1995; Guagliardo, Huang \& D’Angelo, 1999; Herrenkohl et al., 1998; Krohn, Lizotte \& Perez, 1997; Scaramella et al., 1998; Thornberry, Smith \& Howard, 1997). Estes investigam a história de vida destes adolescentes, através de técnicas variadas, a fim de melhor entender a relação entre um fator específico e a paternidade destes jovens. Contudo, suas conclusões indicam que o fenômenoé complexo, sendo influenciado por diversos fatores, nas mais diversas intensidades.

Também é interessante notar que os estudos baseados em dados de pesquisas longitudinais não indicam que tais pesquisas tiveram continuidade após a gestação adolescente. Essa evidência confirmaria a hipótese de que o foco seria apenas ou predominantemente a prevenção do problema, e não a assessoria a estes jovens em sua nova experiência como pais, a fim de que desempenhem seu papel de forma efetiva. Conforme menciona Fonseca (1998), há um silêncio da sociedade em relação à paternidade adolescente, uma espécie de anulação social, uma vez que esta não oferece uma estrutura apropriada para o exercício da paternidade pelo adolescente, o que dificultaria a ele assumir seu papel de pai. Russell (1980) e Montmayor (1986) também constataram uma organização social inadequada para apoiar jovens que se tornam pais na adolescência, por estar esse evento fora do tempo e da seqüência de desenvolvimento considerados social e psicologicamente apropriados.

Sabe-se da importância de um trabalho preventivo. Contudo, um foco tão forte na prevenção poderia estar indicando um pré-conceito, no sentido de que a paternidade adolescente, por ser apenas indesejável, ou uma experiência de consequiências totalmente negativas para os envolvidos, de- 
veria ser evitada a qualquer custo. Não parece haver uma preocupação maior em ajudar estes pais a perceberem aspectos positivos dessa transição, a tirarem proveito de sua experiência, enfim, em auxiliá-los a serem pais efetivos.

\section{Conseqüências/Resultados}

Nesta categoria, todos os estudos estão baseados em enquetes (surveys), de caráter longitudinal (Buchanan \& Robbins, 1990; Christmon \& Luckey, 1994; Heath, McKenry \& Leigh, 1995; Israel \& Seeborg, 1998; Massat, 1995; Nock, 1998; Pirog-Good, 1996) enfatizando dados demográficos dos participantes.

Dentre as conseqüências da paternidade adolescente citadas, encontram-se as econômicas, sociais, educacionais e emocionais (Buchanan \& Robbins, 1990; Heath, McKenry \& Leigh, 1995; Nock, 1998; Pirog-Good, 1996), isoladamente ou conjuntamente. Alguns artigos enfatizam a relação entre a paternidade adolescente e maus tratos infantis (Massat, 1995) ou uso de álcool ou drogas (Christmon \& Luckey, 1994). Apenas um resumo aborda diferenças entre participantes de etnias distintas, encontrando entre os homens negros uma ausência de efeitos psicológicos adversos de longo prazo em função da paternidade na adolescência, o que não ocorreu entre os participantes brancos (Buchanan \& Robbins, 1990).

É interessante ressaltar que a maioria destes estudos tem caráter retrospectivo, isto é, baseia-se em dados de pesquisas realizadas em anos anteriores, principalmente na década de 80 . Nota-se a ausência de um estudo de caráter mais atual. É óbvia a importância de estudos longitudinais que acompanhem estes pais durante vários anos, para retratar de forma completa as conseqüências na vida adulta da paternidade adolescente. Contudo, também é preciso entender quais as conseqüências mais imediatas deste evento sobre o adolescente.

Quanto aos instrumentos, o uso de questionários e a realização de enquetes acarreta maior praticidade para estudos que cobrem grandes períodos de tempo. Contudo, outros instrumentos poderiam ser empregados, tais como entrevista e observação, a fim de que tais informações pudessem ser enriquecidas.

Convém ressaltar que nas conclusões destes estudos percebe-se uma tendência negativa no que diz respeito às conseqüências da paternidade adolescente. Esta condição parece colocar os adolescentes em uma situação de prejuízo nos mais diversos domínios de sua vida. Eles obteriam menor realização acadêmica, alcançariam um nível sócio-econômico inferior, vivenciariam estresses variados etc. Contudo, tais resultados poderiam ser revertidos, como indicam alguns estudos. Um suporte adequado poderia tornar tais consequiências menos negativas, ou até mesmo poderia compensar estes prejuízos sócio-econômicos. Dessa forma, vê-se a ne- cessidade de continuar investigando a vida adulta de pais adolescentes, a fim de se obter dados mais conclusivos e específicos para cada grupo étnico, social etc. Somente desse modo se poderá auxiliar de forma mais efetiva estes pais, criando redes de suporte social que sanassem, ao menos parcialmente, os prejuízos de uma gestação não planejada.

\section{Aspectos familiares/conjugais}

Os estudos contemplados nesta seção enfocam a relação entre comportamentos e/ou sentimentos de pais adolescentes e sua bagagem familiar, ressaltando a questão da transmissão transgeracional de valores e atitudes (Christmon, 1990c; Dellmann-Jenkins, Sattler \& Richardson, 1993; Hardy, Astone, Brooks-Gunn, Shapiro \& Miller, 1998). Tais estudos investigam ainda a influência de membros da família de origem do próprio adolescente e/ou da família da mãe adolescente sobre seu envolvimento com o bebê (Cervera, 1991; Pirog-Good, 1995; Rhein et al., 1997), bem como sobre a relação conjugal com a adolescente (Cutrona, Hessling, Bacon \& Russell, 1998). Um deles também ressalta as consequiências de diferenças étnicas e de idade entre a mãe adolescente e o parceiro (Fernandez, Ruch-Ross \& Montague, 1993). Outro artigo ressalta dados demográficos sobre as uniões de adolescentes em Buenos Aires (Girard, Coll \& Becco, 1991b). Apenas um artigo retrata a vida familiar adulta de pais adolescentes (Heath \& McKenry, 1993). Alguns estudos têm caráter longitudinal (Cervera, 1991; Cutrona et al., 1998; Fernandez, Ruch-Ross \& Montague, 1993; Hardy et al., 1998; Pirog-Good, 1995).

Constata-se que em sua maioria os artigos tratam de aspectos da família de origem do pai adolescente, existindo um pequeno número referente a aspectos conjugais. Além disso, a incidência de artigos sobre o tema não é alta. Esse resultado poderia estar retratando uma concepção de que poucos pais adolescentes se casam com suas parceiras, ou que quando o fazem, a união não tem sucesso. Isso faria com que a união conjugal na adolescência não merecesse grande atenção acadêmica. Contudo, é necessário um questionamento, pois a realidade não parece se apresentar somente dessa forma. Além disso, qualquer tipo de união de adolescentes mereceria ser melhor investigada. Em se tratando do tema, os próprios achados destes estudos indicam que estes pais necessitam de apoio, e que quando presentes, contribuem positivamente para o desenvolvimento de seu bebê e de sua parceira.

Outro ponto a ser ressaltado é a necessidade de mais estudos sobre aspectos familiares, uma vez que os existentes indicam que o apoio familiar é um fator de grande importância no efetivo desempenho do papel paterno pelo adolescente. Conhecendo sua história familiar, seria possível obter um melhor entendimento do contex to em que se insere esta gestação, e de como este adolescente poderá exercer sua 
paternidade. Além disso, o apoio à família de origem de ambos os envolvidos (pai e mãe adolescente) torna-se um fator relevante, pois reverte em benefícios a todos. Estudos longitudinais pareceriam bastante adequados a este objetivo.

Também deve ser ressaltado que estudos transgeracionais devem ser continuamente realizados, uma vez que a estrutura e configuração familiares mudam ao longo do tempo, com novos modelos de família emergindo em nossos dias. Assim, um padrão familiar encontrado em 1990 como favorecedor para uma paternidade adolescente não necessariamente será encontrado nas próximas décadas.

\section{Experiências/Percepções (Atuais elou imaginadas)}

Nesta categoria, a grande maioria dos estudos utiliza entrevistas (individuais ou grupais), a fim de captar sentimentos, percepções e/ou vivências de futuros pais e/ou pais adolescentes. Esta é justamente a diferença entre os resumos classificados nesta categoria ou na categoria "Conseqüências/Resultados". Na categoria anterior, as consequiências são mais específicas e a longo prazo. Aqui os resumos centram-se mais em percepções e os estudos possuem caráter qualitativo.

Os resumos trazem estudos desenvolvidos principalmente com participantes negros (Allen \& Doherty, 1996; Christmon, 1990a; Christmon, 1990b; Dallas \& Chen, 1998; Harris, 1998; Miller, 1994). A percepção das responsabilidades da paternidade pelos pais adolescentes são enfatizadas em alguns estudos (Allen \& Doherty, 1996; Christmon, 1990a; Christmon, 1990b; Nesmith, Klerman, Oh \& Feinstein, 1997). A experiência da paternidade na adolescência também é investigada de forma mais global por alguns estudos (Dallas \& Chen, 1998; Nesmith et al., 1997). Dois resumos enfocam decisões de futuros pais adolescentes encarcerados em relação à gestação (Anderson, 1990; Nesmith et al., 1997). Outros artigos examinam percepções de pais adolescentes sobre temas variados, como sexo, amor, gravidez, paternidade etc. (Harris, 1998; Robinson \& Frank, 1994).

São feitas algumas comparações entre mães e pais adolescentes (Anderson, 1990; Harris, 1998; Robinson \& Frank, 1994), ou entre adolescentes pais e não-pais (Nesmith et al., 1997; Robinson \& Frank, 1994) em alguns artigos.

Dois artigos comentam um artigo anterior que tratava dos efeitos do envolvimento paterno nos resultados intelectuais e comportamentais de seus filhos pré-termos de baixo-peso ao nascer (Kaplan, 1996; Yogman, Kindlon \& Earls, 1996).

Dentre os resultados apontados pelos estudos, em geral nota-se um desejo dos adolescentes de serem pais efetivos, apesar de barreiras percebidas para o seu envolvimento nos mais diversos aspectos. O papel da família de origem foi ressaltado, no sentido dos modelos de relação proporcionados principalmente pelo próprio pai, em geral negativos. $\mathrm{O}$ exercício efetivo da paternidade parece relacionar-se com o próprio desejo de ser um pai efetivo, bem como com as atitudes em relação à família de origem e da parceira, à sexualidade e estratégias de coping utilizadas. Dessa forma, estes resultados indicariam uma tendência positiva no que diz respeito ao envolvimento de pais adolescentes, que iria contra a tendência comumente aceita de que estes pais abandonam suas parceiras e/ou não querem se envolver com elas ou seu bebê devido à pouca idade. Pode-se considerar que os participantes desses estudos constituiriam por si só um grupo diferenciado de adolescentes. Contudo, indicariam talvez uma nova tendência em relação a este evento, que deve ser investigada, pois estas mudanças de atitude deveriam ser acompanhadas por mudanças teóricas e práticas (intervenção). Além disso, retratam o perigo dos preconceitos comumente veiculados em relação aos pais adolescentes. Faz-se necessário pensar se esta visão negativa hegemônica não estaria contribuindo também para minar seu desejo e seu potencial para o envolvimento com o bebê e a parceira (Fonseca, 1998).

\section{Intervenções/Aconselhamento}

Esta categoria assemelha-se muito à categoria revisão da literatura, porém trata de programas ou serviços especializados para pais e gestantes adolescentes, implementados em diversas cidades, principalmente norteamericanas, com uma abordagem psicoeducativa.

Nota-se principalmente que os resumos versam sobre uma avaliação quanto aos resultados alcançados por diversos programas/serviços/intervenções, com descrições do mesmo e/ou dos instrumentos empregados nestes (Hans, Bernstein \& Percansky, 1991; Kiselica, Rotzien \& Doms, 1994; Kost, 1997; Lagges \& Gordon, 1999; Marsh \& Wirick, 1991; Showers, 1991). Os métodos citados são os mais diversos: cartões informativos, $\mathrm{CD}$, manuais.

Nesta categoria os resumos também descrevem percepções e pré-conceitos de profissionais que trabalham com pais e gestantes adolescentes, e as repercussões disto em seu trabalho (Kiselica, Gorcynski \& Capps, 1998; Softas-Nall, Baldo \& Williams, 1997), além de conhecimentos necessários a estes profissionais (Kiselica \& Scheckel, 1995).

Dois artigos referem-se a populações de diferente etnias (Showers, 1992; Softas-Nall, Baldo \& Williams, 1997), sendo que os demais não descrevem com clareza os participantes.

Nota-se que, em geral, os resultados indicam ganhos para pais adolescentes que participam destas intervenções, no sentido de aumento de conhecimentos sobre o desenvolvimento infantil, práticas educativas, métodos contraceptivos, parentalidade etc. De forma mais ampla, isso indicaria uma mudança positiva de atitude destes pais adolescentes, no sentido de exercerem a paternidade de modo efetivo.

Dentre os profissionais, nota-se que estes percebem uma necessidade de mais intervenções visando os pais adoles- 
centes. Além disso, não parecem apresentar conhecimento adequado sobre as necessidades destes pais. Esses resultados parecem corroborar os comentários feitos anteriormente, no sentido da ineficiência das intervenções destinadas a pais adolescentes. Quando nem mesmo os profissionais que lidam com estes jovens sentem-se preparados para isso, a eficácia de qualquer intervenção ficará certamente comprometida. Não se pode também esquecer que estas intervenções muitas vezes abordam o tema da sexualidade e da prevenção da gestação, assuntos considerados ainda como tabus em nossa sociedade. Muitos profissionais sentem grande dificuldade de dialogar sobre este tema, além de possuírem também pré-conceitos em relação à sexualidade adolescente (Kiselica \& Pfaller, 1993; Mackey \& Milloy, 1974). Assim sendo, a intervenção ficaria ainda mais comprometida.

Há uma grande falta de conhecimento sobre o fenômeno da paternidade na adolescência em suas mais diversas formas e efeitos. Essa lacuna teórica poderia estar impedindo que as intervenções e serviços existentes alcancem seus objetivos. Assim, cada vez mais se torna necessária a realização de pesquisas sobre o tema da paternidade adolescente, além de aprimoramento na formação de profissionais que lidam com este evento.

\section{Outros}

Nesta categoria encontram-se artigos que investigam os mesmos fatores antes e durante a paternidade adolescente, sendo considerados como causa e conseqüência desta, simultaneamente. Dentre os fatores estudados, temos: problemas sexuais (Ketterlinus, Lamb, Nitz \& Elster, 1992) e comportamento anti-social/delinquiência (Fagot, Pears, Capaldi, Crosby \& Leve, 1998; Florsheim, Moore, Zollinger, McDonald \& Sumida, 1999; Stouthamer-Loeber \& Wei, 1998). Além disso, os estudos têm caráter longitudinal em sua maioria (Fagot et al.,1998; Florsheim et al., 1999; Stouthamer-Loeber \& Wei, 1998). As conclusões destes estudos apontam para algumas relações existentes entre estes fatores e a paternidade adolescente, ressaltando sempre, contudo, a necessidade de estudos que abranjam o ambiente mais amplo, a fim de se estabelecer também alguns fatores intervenientes. Assim, vê-se a importância de englobar nos estudos questões sociais e culturais, uma vez que estas enriquecem o entendimento do evento.

Percebe-se, ao longo dos anos, uma certa mudança no enfoque das pesquisas. Antes, artigos focalizavam principalmente as causas ou as consequiências da paternidade adolescente. Atualmente, uma tendência nova aparece: investigar o mesmo fator de risco antes e durante a paternidade, a fim de verificar seu potencial de influência como causa e como determinante do tipo de paternidade exercida. Podese pensar que esse enfoque enriquece as pesquisas, pois na verdade fatores isolados não deveriam ser considerados ape- nas causas da paternidade adolescente, já que também produzem certas conseqüências, muitas vezes a própria repetição do evento. Por exemplo, maus tratos na infância parecem estar relacionados à paternidade adolescente, e ao mesmo tempo pais adolescentes podem maltratar seus filhos (Fagot et al., 1998).

Na terceira etapa, os resumos também foram classificados em relação a alguns aspectos metodológicos, tais como características dos participantes, o delineamento empregado, os instrumentos utilizados e o tipo de análise de dados. Optou-se por excluir desta análise resumos de estudos de revisão da literatura, por não indicarem detalhadamente estas informações. É interessante ressaltar que alguns resumos classificados em outros tópicos também não continham estes dados, por isso as frequiências encontradas, expostas nos gráficos, não correspondem ao número total de artigos (68). Além disso, determinados artigos foram classificados simultaneamente em alguns itens. Os resultados encontrados estão expostos nos gráficos.

Em relação aos participantes destes estudos, os resultados encontram-se na Figura 4. Percebe-se uma forte tendência étnica, tendo alguns estudos sido realizados somente com participantes negros (Allen \& Doherty, 1996; Christmon, 1990a, 1990b, 1990c; Dallas \& Chen, 1999; Harris, 1998; Miller, 1994), ou utilizando comparações entre diversos grupos étnicos (por exemplo, Buchanan \& Robbins, 1990; Fernandez, Ruch \& Montague, 1993; Guagliardo, Huang \& D'Angelo, 1999; Nesmith, Klerman, Oh \& Feinstein, 1997; Pirog-Good, 1995; Robinson \& Frank, 1994; Showers, 1992), inclusive o negro. Nota-se esta tendência nos artigos norte-americanos, que talvez pudesse ser explicada pela maior incidência de gravidez adolescente entre a população negra naquele país, que desfruta ainda de um padrão sócioeconômico mais baixo que o da população branca, por exemplo. Contudo, denota certo preconceito, pois adolescentes brancas também engravidam, mesmo que estes números não sejam divulgados em virtude das medidas tomadas em classes mais elevadas. Ao mesmo tempo, se poderia pensar que o acesso dos pesquisadores à população negra é mais fácil, até pela sua dependência em relação aos serviços públicos. De qualquer forma, felizmente se percebe uma diminuição no número destes estudos ao longo do tempo, o que poderia indicar uma nova tendência nas pesquisas, que atualmente privilegiam comparações entre pais e mães adolescentes, uma vez que as experiências vividas por pais de etnias diversas não se mostraramtão diferentes quanto poderiam parecer inicialmente.

As comparações entre gênero, também bastante enfatizadas, ocorrem entre a gestante adolescente e o futuro pai adolescente (por exemplo, Dellmann-Jenkins, Sattler \& Richardson, 1993; Fernandez, Ruch \& Montague, 1993; Florsheim et al., 1999; Harris, 1998; Herrenkohl et al., 1998; 
Israel \& Seeborg, 1998; Ketterlinus et al., 1992; Lagges \& Gordon, 1999; Nock, 1998; Rhein et al., 1997; Robinson \& Frank, 1994; Showers, 1991). Visam investigar principalmente suas diferenças em diversos aspectos, como no caso das necessidades que apresentam. O intuito é sempre o de mostrar caminhos para intervenções futuras, a fim de melhor atendê-los. Os resultados indicam que a gravidez na adolescência produz um grande impacto na vida de adolescentes de ambos os sexos, e inclusive percebe-se um maior prejuízo para o pai adolescente no sentido da escolarização e da responsabilidade financeira. Assim, estes resultados não corresponderiam à idéia socialmente veiculada de que as mães adolescentes seriam as maiores vítimas deste evento. Ambos sofreriam com a gravidez precoce, apenas de forma diferenciada.

Comparações entre os pais adolescentes e suas parceiras são importantes, por ressaltarem suas necessidades específicas. Percebe-se contudo que os pais parecem ainda se encontrar em estado de desvantagem quando comparados às mães adolescentes no que diz respeito ao atendimento de suas necessidades.

As comparações entre estar esperando um filho ou não, são bastante freqüentes (por exemplo, Anderson, 1990; Buchanan \& Robbins, 1990; Christmon \& Luckey, 1994; Dearden, Hale \& Alvarez, 1992; Dearden, Hale \& Woolley, 1995; Fagot et al., 1998; Herrenkohl et al., 1998; Israel \& Seeborg, 1998; Nesmith et al., 1997; Pirog-Good, 1995; Robinson \& Frank, 1994; Stouthamer-Loeber \& Wei, 1998;
Thornberry, Smith \& Howard, 1997). Visam verificar a influência do fator paternidade sobre diferentes variáveis, tais como deliqüencia, consequiências sócio-econômicas, desempenho escolar, dentre outras.

As comparações por idade são realizadas entre jovens pais e pais adultos (por exemplo, Cervera, 1991; Dearden, Hale \& Woolley, 1995; Girard, Coll \& Becco, 1991a; Heath, McKenry \& Leigh, 1995; Kessler et al., 1997; Ketterlinus et al., 1992; Robinson \& Frank, 1994; Showers, 1992). No entanto, pouco se investiga sobre os aspectos mais subjetivos, como sentimentos, percepções, vivências entre os grupos. As análises centram-se em diferenças no status sócioeconômico.

Em geral, os estudos não parecem levar em conta comparações entre grupos de nível sócio-econômico variado (baixo e médio). Apenas um estudo foi encontrado utilizando tal comparação (Florsheim et al., 1999). Contudo, este aspecto se torna importante, ainda mais quando as estatísticas demonstram que o maior número de gestação na adolescência encontra-se ainda na classe sócio-econômica baixa.

A partir da Figura 5, pode-se constatar que o instrumento mais utilizado em pesquisas com pais adolescentes é o questionário (por exemplo, Buchanan \& Robbins, 1990; Cervera, 1991; Christmon, 1990a; 1990b, 1990c; Christmon \& Luckey, 1994; Dearden, Hale \& Alvarez, 1992; Dearden, Hale \& Woolley, 1995; Dellmann-Jenkins, Sattler \& Richardson, 1993; Girard, Coll \& Becco, 1991a; Guagliardo,

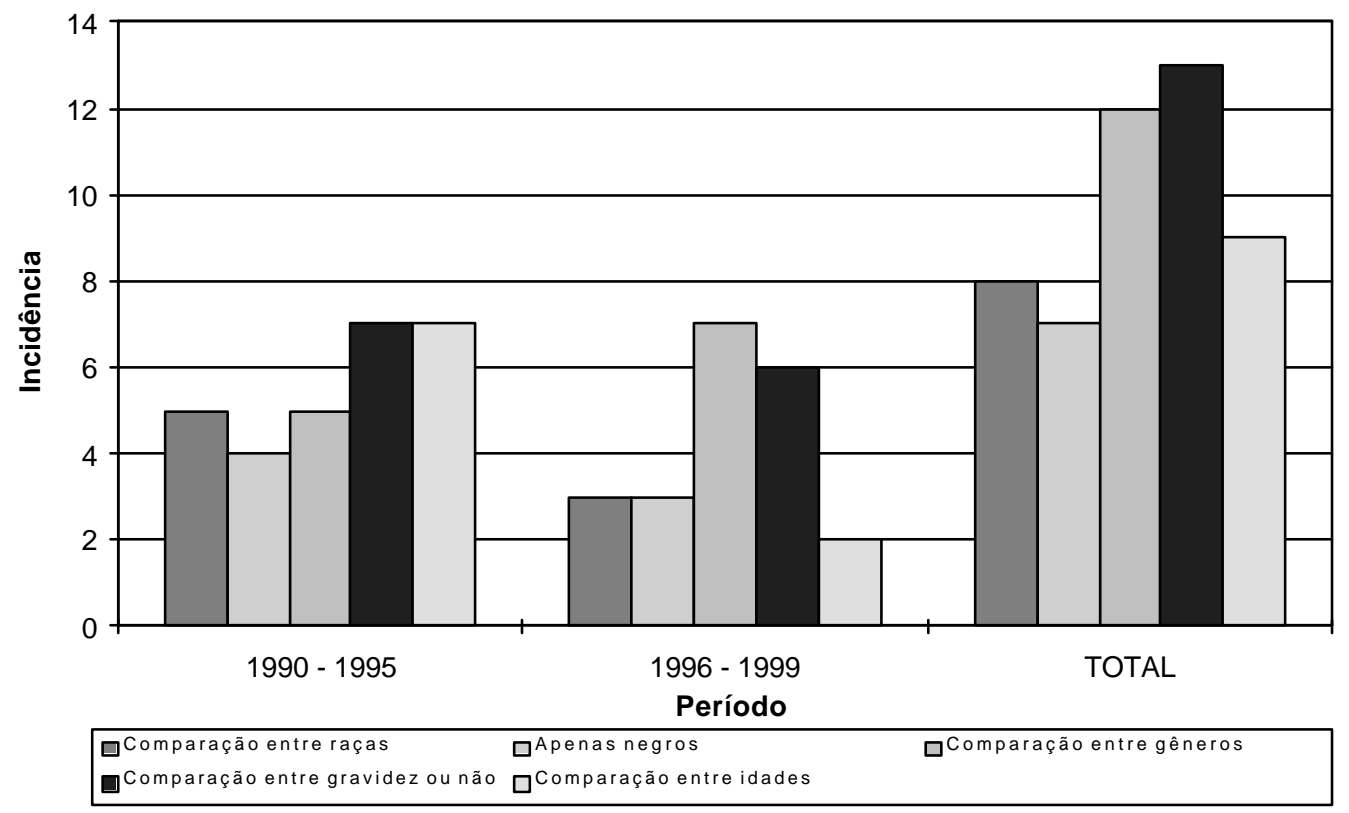

Figura 4. Incidência de estudos sobre paternidade adolescente, de acordo com os participantes e o período de consulta ao Psyclit 
Huang \& D’Angelo, 1999; Harris, 1998; Israel \& Seebrog, 1998; Ketterlinus et al., 1992; Lagges \& Gordon, 1999; Miller, 1994; Nock, 19998; Heath \& McKenry, 1993; Heath, McKenry \& Leigh, 1995; Pirog-Goog, 1995; Pirog-Good, 1996; Showers, 1992; Stouthamer-Loeber \& Wei, 1998; Thornberry, Smith \& Howard, 1997). Seria possível supor que a grande utilização desse instrumento de pesquisa se deve ao tipo de população estudada. A participação de um adolescente seria facilitada pela aplicação de um questionário rápido, impessoal, anônimo. Além disso, a maioria dos estudos que utilizou questionário é de caráter longitudinal. Assim, conforme já exposto anteriormente, devido à grande duração destes estudos, o questionário se torna um instrumento adequado, por ser rápido, econômico, acessível. No entanto, a aplicação de um questionário não assegura a resposta honesta do adolescente a todas as questões, ou até mesmo que responda a todas as questões.

Vê-se também que a entrevista é a segunda técnica mais utilizada, tendo seu uso mais difundido nos estudos recentes (por exemplo, Allen \& Doherty, 1996; Anderson, 1990; Cervera, 1991; Kessler et al., 1997; Nesmith et al., 1997; Rhein et al., 1997). Não seria tão impossível considerar esse uso como uma resposta à grande utilização de questionários em estudos de anos anteriores. A entrevista poderia estar vindo ampliar os dados dos questionários, pois é utilizada na maioria dos estudos qualitativos, a fim de investigar percepções, sentimentos e vivências de pais adolescentes.

Chama a atenção o pequeno número de estudos utilizando grupos (por exemplo, Dallas \& Chen, 1998; Harris,
1998; Lagges \& Gordon, 1999), o que parece ser uma tendência nova, presente nos estudos qualitativos. Além disso, a observação também é muito pouco utilizada (por exemplo, Cutrona et al., 1998; Fagot et al., 1998; Lagges \& Gordon, 1999), e em nenhum momento foi utilizada observação da interação entre pais adolescentes e seus bebês. A observação apareceu somente em intervenções, em que os participantes observaram vídeos preventivos (por exemplo, Lagges \& Gordon, 1999).

A inexistência de estudos utilizando testes também é um fato marcante. Talvez testes pudessem ser acrescentados a outros instrumentos, a fim de ampliar o entendimento de fatores intrapsíquicos e sua influência no modo como o adolescente lida com a paternidade.

Quanto ao delineamento utilizado (Figura 6), surpreende a incidência superior de estudos longitudinais (por exemplo, Anderson, 1990; Buchanan \& Robbins, 1990; Cervera, 1991; Cutrona et al., 1998; Dearden, Hale \& Alvarez, 1992; Dearden, Hale \& Woolley, 1995; Fagot et al., 1998; Florsheim et al., 1999; Harris, 1998; Heath \& McKenry, 1993; Heath, McKenry \& Leigh, 1995; Herrenkohl et al., 1998; Israel \& Seeborg, 1998; Kessler et al., 1997; Lagges \& Gordon, 1999; Nock, 1998; Pirog-Good, 1995, 1996; Showers, 1992; Stouthamer-Loeber \& Wei, 1998; Thornberry, Smith \& Howard, 1997). Em sua maioria, estes estudos são bastante amplos, iniciados principalmente nos anos 80, nos EUA e Inglaterra. Os dados são utilizados retrospectivamente. Percebe-se que estes estudos apresentam um delineamento adequado, no momento que muitos inves-

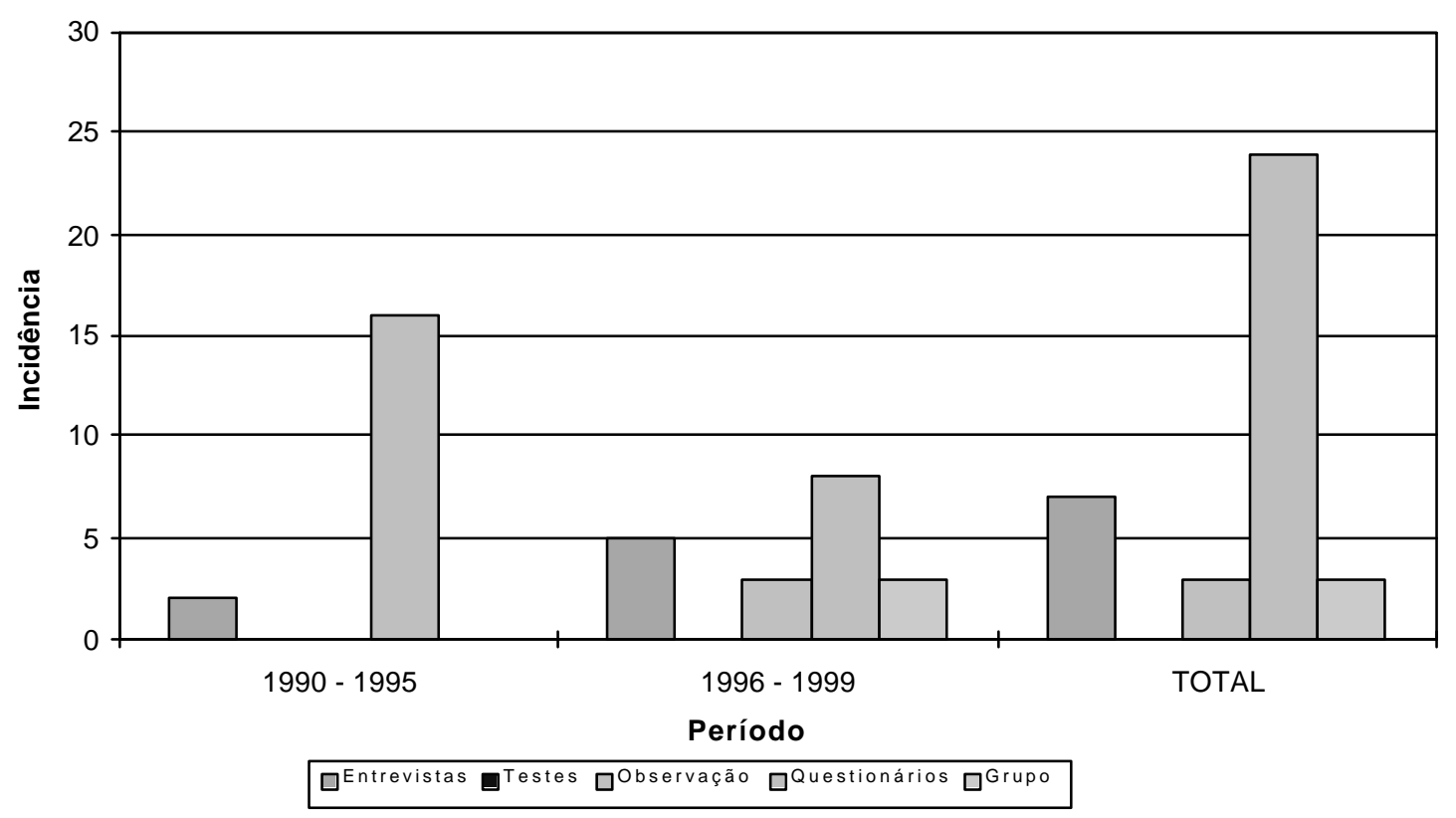

Figura 5. Incidência de estudos sobre paternidade adolescente, de acordo com instrumentos utilizados e o período de consulta ao Psyclit 
tigam fatores anteriores e posteriores à gestação adolescente, conforme citado anteriormente. Contudo, percebe-se a existência de poucos estudos que cubram uma menor extensão de tempo, investigando mais profundamente as experiências mais imediatas de pais adolescentes (por exemplo, Allen \& Doherty, 1996; Christmon, 1990a; 1990b, 1990c; Christmon \& Luckey, 1994; Dellmann-Jenkins, Sattler \& Richardson, 1993; Fernandez, Ruch \& Montague, 1993; Girard, Coll \& Becco, 1991a; Guagliardo, Huang \& D’Angelo, 1999; Ketterlinus et al., 1992; Miller, 1994; Nesmith et al., 1997; Rhein et al., 1997; Robinson \& Frank, 1994).

Já no que diz respeito à análise dos dados (Figura 7), é necessário ressaltar que este tópico foi o menos explicitado nos resumos. Assim, muitos foram excluídos por não apresentarem este tipo de informação.

Dentre os resumos considerados, é gritante a diferença entre a incidência de estudos que utilizaram análise qualitativa (por exemplo, Anderson, 1990; Allen \& Doherty, 1996; Dallas \& Chen, 1998; Harris, 1998; Nesmith et al., 1997) e quantitativa dos dados (por exemplo, Buchanan \& Robbins, 1990; Cervera, 1991; Christmon, 1990a; 1990b, 1990c; Christmon \& Luckey, 1994; Dellmann-Jenkins, Sattler \& Richardson, 1993; Dearden, Hale \& Alvarez, 1992; Dearden, Hale \& Woolley, 1995; Fagot et al., 1998; Fernandez, Ruch \& Montague, 1993; Florsheim et al., 1999; Girard, Coll \& Becco, 1991a; Guagliardo, Huang \& D’Angelo, 1999; Harris, 1998; Heath \& McKenry, 1993; Heath, McKenry \& Leigh, 1995; Herrenkohl et al., 1998; Israel \& Seeborg, 1998;
Lagges \& Gordon, 1999; Kessler et al., 1997; Ketterlinus et al., 1992; Miller, 1994; Nesmith et al., 1997; Nock, 1998; Pirog-Good, 1995, 1996; Rhein et al., 1997; Robinson \& Frank, 1994; Showers, 1991; Stouthamer-Loeber \& Wei, 1998; Thornberry, Smith \& Howard, 1997), com maior incidência destes últimos. Ainda se percebe a existência de alguns estudos que utilizaram uma metodologia combinada (por exemplo, Harris, 1998). Relacionando-se estes achados com os resultados expostos no gráfico anterior, percebe-se que poderiam estar relacionados à diferença observada entre o número de estudos empregando questionários e entrevistas, por exemplo.

Desde os instrumentos percebe-se uma vertente quantitativa nos estudos sobre a paternidade adolescente. Considerando-se a característica da pesquisa em Psicologia desde seus primórdios, constata-se que o aspecto quantitativo era considerado indispensável para tornar um estudo científico e confiável. Além disso, não se pode esquecer que a grande maioria destes estudos foi realizado nos EUA, um país de tradição quantitativa em pesquisa, onde uma cultura pragmática ainda impera. Assim, os estudos são fortemente influenciados por este viés. Felizmente, percebe-se em alguns estudos, principalmente os mais atuais, o uso de metodologia qualitativa.

Parece, pois, que tais estudos quantitativos não estavam fornecendo os conhecimentos necessários para gerar intervenções eficazes sobre este evento. Estudos qualitativos deveriam ainda ser realizados em maior número, a fim de prover dados sobre aspectos subjetivos, que influenciam

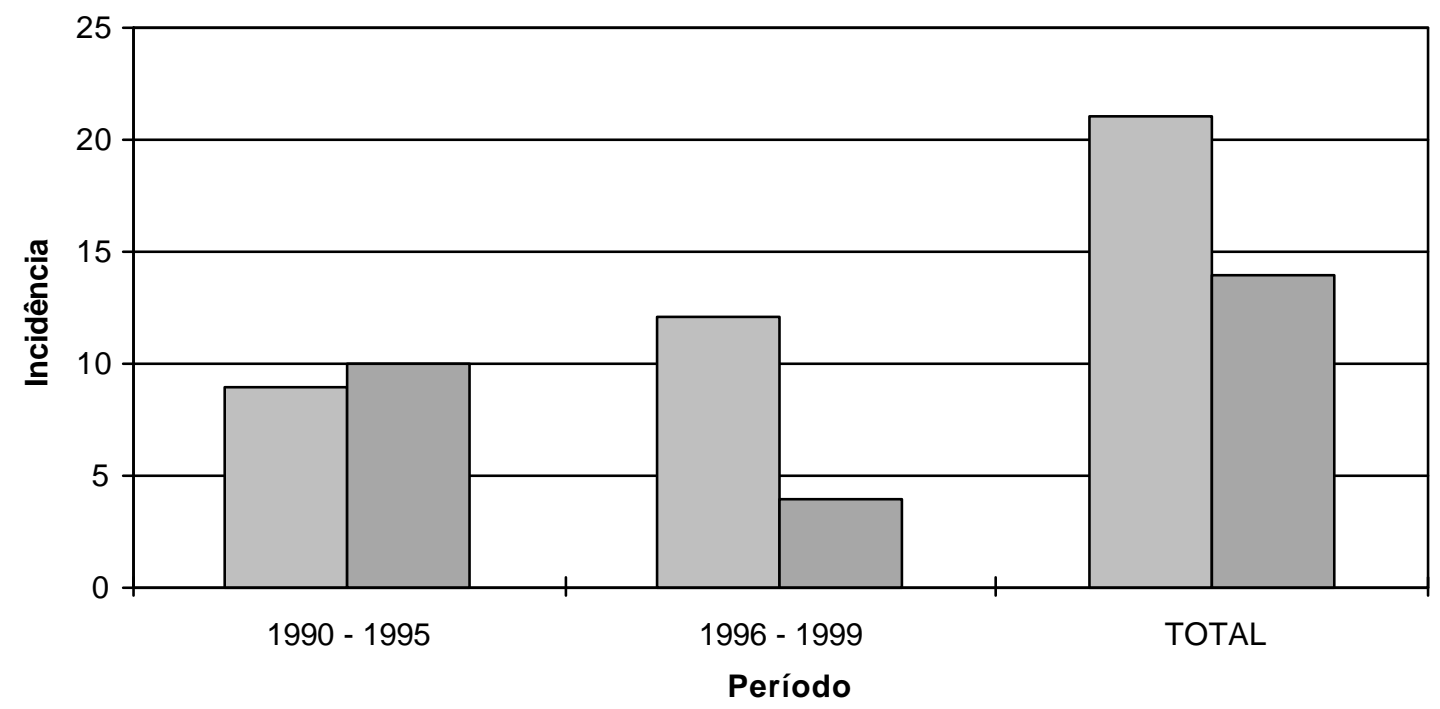

$\square$ Estudos longitudinais $\square$ Estudos transversais

Figura 6. Incidência de estudos sobre paternidade adolescente, de acordo com o delineamento utilizado e o período de consulta ao Psyclit 


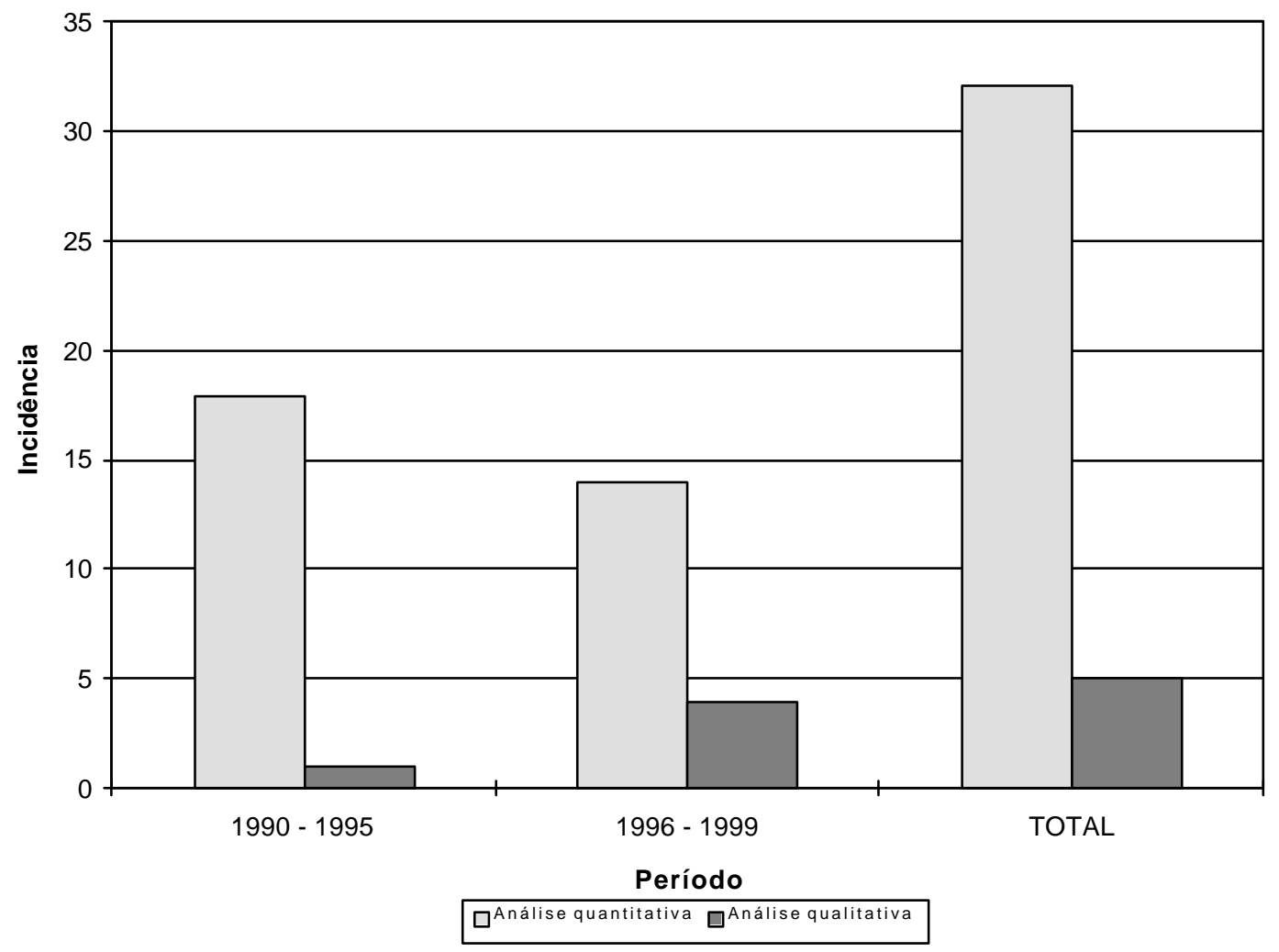

Figura 7. Incidência de estudos sobre paternidade adolescente, de acordo com o tipo de análise de dados e o período de consulta ao Psyclit

fortemente o modo como o pai adolescente irá reagir a qualquer tipo de intervenção ou aconselhamento. Além disso, conhecendo-se as percepções, sentimentos e vivências destes jovens, pode-se talvez produzir um conhecimento mais consistente e até menos preconceituoso a seu respeito.

\section{Conclusões}

Ao final deste trabalho, constata-se a relevância da realização de um estudo de revisão da literatura. Este permite uma visão rápida e ao mesmo tempo abrangente da produção científica de determinada época sobre um tema específico. A partir de tal visão, pode-se direcionar esforços de pesquisa para áreas pouco exploradas, evitando a mesmice e possibilitando maior avanço nos conhecimentos já existentes sobre o tema em questão (Piccinini \& Lopes, 1994).

No caso da paternidade adolescente, nota-se a necessidade de realização de estudos que investiguem principalmente aspectos subjetivos de futuros pais e/ou pais adolescentes, tais como suas expectativas, sentimentos, percepções e vivências, seja sobre si mesmos e/ou sobre seu bebê. Além disso, também se faz necessário estudar de forma mais detalhada a relação conjugal e com a família de origem destes jovens, bem como sua relação com seu bebê. No que diz respeito aos instrumentos, a observação, os testes e a entre- vista poderiam ser aplicados de forma mais sistemática. Os estudos também poderiam estar mais centrados na experiência atual de pais adolescentes, em vez de centrar-se em dados retrospectivos e terem longa duração.

Outro ponto importante a ser ressaltado refere-se à organização dos resumos. Mesmo em uma base de dados como o Psyclit, nota-se a ausência de muitas informações, tais como objetivos do estudo, participantes, metodologia empregada e inclusive resultados. Seria importante que tais aspectos recebessem mais atenção dos pesquisadores. Este tipo de problema não aparece apenas nos estudos sobre a paternidade adolescente. Piccinini e Lopes (1994) encontraram resultados semelhantes em sua investigação sobre artigos publicados na área da psicologia infantil.

Os resultados encontrados motivaram a continuação do estudo, a fim de abarcar agora a realidade acadêmica nacional sobre o tema da paternidade adolescente. Foi realizada uma consulta inicial à nova base de dados organizada pelo Conselho Federal de Psicologia. Os resultados encontrados apontam na mesma direção, como mostra a Tabela 1.

Assim, percebe-se que a realidade nacional se assemelha muito à internacional. Parece que a paternidade adolescente não existe como objeto de pesquisa no meio acadêmico brasileiro. Felizmente, sabe-se da existência de alguns 
Tabela 1.

Incidência de estudos nacionais sobre a paternidade e a maternidade

Termo

Total

Mãe

253

Pai

106

Maternidade

Paternidade

Maternidade adolescente

Paternidade adolescente

trabalhos recentes que não constam desta base de dados, conduzidos na USP/Ribeirão Preto, sob orientação da Profa. Maria Alves de Toledo Bruns, e na UFPE, sob orientação do Prof. Jorge Lyra. Este último criou inclusive o programa PAPAI, destinado somente ao estudo da paternidade.

Entretanto, percebe-se que o tema da paternidade adolescente parece ser apenas novidade tanto internacionalmente como no Brasil, apesar do grande número de gestações adolescentes apontadas pelas estatísticas a cada ano. Sem dúvi$\mathrm{da}$, este evento apresenta-se como um tema de pesquisa bastante rico, que merece ser aprofundado. Os achados desta revisão apontam diversos caminhos, que precisam apenas de pesquisadores interessados e curiosos para serem trilhados.

\section{Referências}

Dimenstein, G. (1999, 15 de maio). Desinformação provoca riscos sexuais. Folha de São Paulo, Seção Cotidiano, p. 5.

Elster, A. B., \& Lamb, M. E. (Org.). (1986). Adolescent fatherhood. Hillsdale: Lawrence Erlbaum.
Fonseca, J. L. C. L. (1998). Paternidade adolescente: da investigação à intervenção. In M. Arilha, S. G. U. Ridenti \& B. Medrado (Orgs.), Homens e masculinidades: outras palavras (pp. 185 - 214). São Paulo: Editora 34.

Hendricks, L. E., \& Montgomery, T. (1983). A limited population of unmarried adolescent fathers: A preliminary report of their views on fatherhood and the relationship with the mothers of their children. Adolescence, XVIII (69), 201-210.

Kiselica, M. S., \& Pfaller, J. (1993). Helping teenage parents: The independent and collaborative roles of counselor educators and school counselors. Journal of Counseling \& Development, 72(1), 42-48.

Mackey, B., \& Milloy, M. (1974). The impact of the teenage pregnancy on the professional educator. The Family Coordinator, 23(1), 15-18.

Montemayor, R. (1986). Boys as fathers: Coping with the dilemmas of adolescence. In A. B. Elster \& M. E. Lamb (Org.), Adolescent fatherhood (pp. 1-18). Hillsdale: Lawrence Erlbaum.

Piccinini, C. A., \& Lopes, R. C. S. (1994). A pesquisa em Psicologia infantil no Brasil: alguns aspectos críticos. Cadernos da ANPEPP, 2 43-55.

Russell, C. S. (1980). Unescheduled parenthood: Transition to 'parent' for the teenager. Journal of Social Issues, 36(1), 45-63).

Soares, I. (1999, 21 de novembro). Gravidez precoce: gestações jovens estão sem controle. Zero Hora, Seção Geral, p. 47-49.

\section{Nota}

1 Trabalho apresentado em Sessão Coordenada no III Congresso Brasileiro de Psicologia do Desenvolvimento, Niterói, 13 a 15 de julho de 2000.

Daniela Centenaro Levandowski, psicóloga (PUCRS), mestre em Psicologia do Desenvolvimento (UFRGS-CNPq), doutoranda em Psicologia do Desenvolvimento (UFRGS), membro do Comitê de Bebês da Sociedade de Psicologia do RS, é docente da Universidade do Oeste de Santa Catarina (UNOESC), Campus de Chapecó. Endereço para correspondência: Rua Ramiro Barcelos, 2600, sala 110, Bairro Rio Branco, 90035-030, Porto Alegre, RS. E-mail: d.cl@zaz.com.br 


\section{Anexo}

Allen, W. D., \& Doherty, W. J. (1996). The responsibilities of fatherhood as perceived by African American teenage fathers. Families in Society: The Journal of Contemporary Human Services, 77(3), 142-155.

Anderson, N. L. (1990). Pregnancy resolution decisions in juvenile detention. Archives of Psychiatric Nursing, 45), 325-331.

Becker, E. L., \& Rickel, A. U. (1995). Integration of teen pregnancy and child abuse research: Identifying mediator variables for pregnancy outcome. Journal of Primary Prevention, 16 (8), 39-53.

Benoit, M. B. (1997). The role of psychological factors on teenagers who become parents out-of-wedlock. Children and Youth Services Review, 19(5-6), 401413.

Buchanan, M., \& Robbins, C. (1990). Early adult psychological consequences for males of adolescent pregnancy and its resolution. North Central Sociological Association Meetings (1988, Pittsburgh, Pennsylvania). Journal of Youth and Adolescence, 19(4), 413-424.

Cervera, N. (1991). Unwed teenage pregnancy: Family relationships with the father of the baby. Families in Society, 72(1), 29-37.

Christmon, K. (1990a). Parental responsibility of African-American unwed adolescent fathers. Adolescence, 25(99), 645-653.

Christmon, K. (1990b). Parental responsibility and self-image of African American fathers. Families in Society, 71(9), 563-567.

Christmon, K. (1990c). The unwed adolescent father's perceptions of his family and of himself as a father. Child and Adolescent Social Work Journal, 7(4), 275-283.

Christmon, K., \& Luckey, I. (1994). Is early fatherhood associated with alcohol and other drug use?. Journal of Substance Abuse, (3), 337-343.

Cochran, D. L. (1997). African American fathers: A decade review of the literature. Families in Society, 78(4), 340-350.

Cutrona, C. E., Hessling, R. M., Bacon, P. L., \& Russell, D. W. (1998). Predictors and correlates of continuing involvement with the baby's father among adolescent mothers. Journal of Family Psychology, 12(3), 369-387.

Dallas, C. M., \& Chen, S. C. (1998). Experiences of African American adolescent fathers. Western Journal of Nursing Research, 20(2), $210-222$.

Dearden, K. A., Hale, C. B., \& Alvarez, J. (1992). The educational antecedents of teen fatherhood. British Journal of Educational Psychology, 62, $139-147$.

Dearden, K. A., Hale, C. B., \& Woolley, T. (1995). The antecedents of teen fatherhood: A retrospective case-control study of Great Britain youth. American Journal of Public Health, 58(4), 551-554.

Dellmann-Jenkins, M., Sattler, S. H., \& Richardson, R. A. (1993). Adolescent parenting: A positive, intergenerational approach. Families in Society, 74(10), 590-601.

Fagot, B. I., Pears, K. C., Capaldi, D. M., Crosby, L., \& Leve, C. S. (1998). Becoming an adolescent father: Precursors and parenting. Developmental Psychology, 34(6), 1209-1219.

Fernandez, M., Ruch-Ross, H. S., \& Montague, A. P. (1993). Ethnicity and effects of age gap between unmarried adolescent mothers and partners. Journal of Adolescent Research, 84), 439-466.

Florsheim, P., Moore, D., Zollinger, L., MacDonald, J., \& Sumida, E. (1999). The transition to parenthood among adolescent fathers and their partners: Does antisocial behavior predict problems in parenting? Applied Developmental Science, 3(3), 178-191.

Girard, G. A., Coll, A., \& Becco, L. (1991a). The adolescent father: Somebody who needs assistance: Implications for health care. International Journal of Adolescent Medicine and Health, 5(2), 127-133.

Girard, G. A., Coll, A., \& Becco, L. (1991b). Characteristics of adolescent fathers in Buenos Aires. International Journal of Adolescent Medicine and Health, 5(2), 135-140.

Guagliardo, M. F., Huang, Z., \& D'Angelo, L. J. (1999). Fathering pregnancies: Marking health-risk behaviors in urban adolescents. Journal of Adolescent Health, 24(1), 10-15.

Hans, S. L., Bernstein, V. J., \& Percansky, C. (1991). Adolescent parenting programs: Assessing parent-infant interaction [Special Issue: Services to teenage parents]. Evaluation and Program Planning, 14(1-2), 87-95.

Hardy, J. B., Astone, N. M., Brooks-Gunn, J., Shapiro, S., \& Miller, T. L. (1998). Like mother, like child: Intergenerational patterns of age at first birth and associations with childhood and adolescent characteristics and adult outcomes in the second generation. Developmental Psychology, 34(6), 1220-1232.

Harris, J. L. J. (1998). Urban African American adolescent parents: Their perceptions of sex, love, intimacy, pregnancy and parenting. Adolescence, 33(132), 833-844.

Heath, D. T., \& McKenry, P. C. (1993). Adult family life of men who fathered as adolescents. Families in society: The Journal of Contemporary Human Services, 74(1), 36-45.

Heath, D. T., McKenry, P. C., \& Leigh, G. K. (1995). The consequences of adolescent parenthood on men's depression, parental satisfaction and fertility in adulthood. Journal of Social Service Research, 20(3-4), 127-148.

Herrenkohl, E. C., Herrenkohl, R. C., Egolf, B. P., \& Russo, M. J. (1998). The relationship between early maltreatment and teenage parenthood. Journal of Adolescence, 21(3), 291-303.

Israel, M., \& Seeborg, M. (1998). The impact of youth characteristics and experiences on transitions out of poverty. Journal of Socio Economics, 27(6), 753776.

Joshi, N. P., \& Battle, S. F. (1990). Adolescent fathers: An approach for intervention. Journal of Health and Social Policy, 13), 17-33.

Kaplan, M. D. (1996). The role of fathers. Journal of the American Academy of Child and Adolescent Psychiatry, 35(6), 699-700.

Kessler, R. C., Berglund, P. A., Foster, C. L., Saunders, W. B., Stang, P. E., \& Walters, E. E. (1997). American Journal of Psychiatry, 154(10), 1405-1411.

Ketterlinus, R. D., Lamb, M. E., Nitz, K., \& Elster, A. B. (1992). Adolescent nonsexual and sex-related problem behaviors. Journal of Adolescent Research, 7(4), 431-456.

Kirby, D. (1991). School-based clinics: Research results and their implications for future research methods [Special Issue: Services to teenage parents]. Evaluation and Program Planning, 14(1-2), 35-47. 
Kiselica, M. S., Stroud, J., Stroud, J., \& Rotzien, A. (1992). Counseling the forgotten client: The teen father. Journal of Mental Health Counseling, 14(3), 338-350.

Kiselica, M. S., \& Pfaller, J. (1993). Helping teenage parents: The independent and collaborative roles of counselor educators and school counselors. Journal of Counseling \& Development, 72(1), 42-48.

Kiselica, M. S., \& Sturmer, P. (1993). Is society giving teenage fathers a mixed message? Youth and Society, 24(4), 487-501.

Kiselica, M. S., \& Murphy, D. K. (1994). Developmental career counseling with teenage parents. Career Development Quarterly, 42(3), $238-243$.

Kiselica, M. S., Rotzien, A., \& Doms, Jill (1994). Preparing teenage fathers for parenthood: A group psychoeducational approach [Special Issue: Counseling men]. Journal of Specialists in Group Work, 19(2), 83-94.

Kiselica, M. S., \& Scheckel, S. (1995). The couvade syndrome (sympathetic pregnancy) and teenage fathers: A brief primer for school counselors. School Counselor, 43(1), 42-51.

Kiselica, M. S., Gorcynski, J., \& Capps, S. (1998). Teen mothers and fathers: School counselor perceptions of service needs. Professional School Counseling, 2(2), 146-152.

Kost, K. (1997). The effects of support on the economic well-being of young fathers. Families in Society, 78(4), 370-382.

Krohn, M. D., Lizotte, A. J., \& Perez, C. M. (1997). The interrelationship between substance use and precocious transitions to adult statuses. Journal of Health and Social Behavior, 38(1), 87-103.

Lagges, A. M., \& Gordon, D. A. (1999). Use of an interactive laserdisc parent training program with teenage parents. Child and Family Behavior Therapy, 2l(1), 19-37.

Marsh, J. C., \& Wirick, M. A. (1991). Evaluation of Hull House teen pregnancy and parenting program [Special Issue: Services to teenage parents]. Evaluation and Program Planning, 14,(1-2), 49-61.

Marsiglio, W. (1995). Young nonresident biological fathers. Marriage and Family Review, 20(3-4), 325-348.

Massat, C. R. (1995). Is older better? Adolescent parenthood and maltreatment. Child Welfare, 74(2), 325-336.

Miller, B. C. (1992). Adolescent parenthood, economic issues, and social polices [Special Issue: Toward the turn of the century: Families and economic realities]. Journal of Family and Economic Issues, 13(4), 467-475.

Miller, D. B. (1994). Influences of parental involvement of African American adolescent fathers. Child and Adolescent Social Work Journal, 11 (5), 363378.

Miller, D. B. (1997). Adolescent fathers: What we know and what we need to know. Child and Adolescent Social Work Journal, 14(1), 55-69.

Moroz, K. J., \& Allen-Meares, P. (1991). Assessing adolescent parent and their infants: Individualized family service planning. Families in Society, 72(8), 461-468.

Nesmith, J. D., Klerman, L. V., Oh, M. K., \& Feinstein, R. A. (1997). Procreative experiences and orientations toward paternity held by incarcerated adolescent males. Journal of Adolescent Health, 20(3), 198-203.

Nock, S. L. (1998). The consequences of premarital fatherhood. American Sociological Review, 63, 250-263.

Pirog-Good, M. A. (1996). The education and labor market outcomes of adolescent fathers. Association for Public Policy Analysis and Management (1992, US). Youth and Society, 28(2), 236-262.

Rhein, L. M., Ginsburg, K. R., Schwarz, D. F., Pinto-Martin, J. A., Zhao, H., Morgan, A. P., \& Slap, G. B. (1997). Teen father participation in child rearing: family perspectives. Journal of Adolescent Health, 21, 244-252.

Robinson, R. B., \& Frank, D. I. (1994). The relation between self-esteem, sexual activity, and pregnancy. Adolescence, 29(113), 27-35.

Roye, C. F., \& Balk, S. J. (1996). The relationship of partner support to outcomes for teenage mothers and their children: A review. Journal of Adolescent Health, 19(2), 86-93.

Scaramella, L. V., Conger, R. D., Simons, R. L., \& Whitbeck, L. B. (1998). Predicting risk for pregnancy by late adolescence: A social contextual perspective. Developmental Psychology, 34(6), 1233-1245.

Serbin, L. A., \& Stack, D. M. (1998). Introduction to the special section: Studying intergenerational continuity and the transfer of risk. Developmental Psychology, 34(6), 1159-1161.

Showers, J. (1991). Child behavior management cards: Prevention tools for teens. Child Abuse and Neglect, 15(3), 313-316.

Showers, J. (1992). "Don't shake the baby": The effectiveness of a prevention program. Child Abuse and Neglect, 16(1), $11-18$.

Softas, B. N., Baldo, T. D., \& Williams, S. C. (1997). Counselor trainee perceptions of Hispanic, Black, and White teenage expectant mothers and fathers. Journal of Multicultural Counseling and Development, 25(4), 234-243.

Stevens-Simon, C., \& Nelligan, D. (1998). Strategies for identifying and treating adolescents at risk for maltreating their children. Aggression and Violent Behavior, 32), 197-217.

Stouthamer-Loeber, M., \& Wei, E. H. (1998). The precursors of young fatherhood and its effect on delinquency of teenage males. Journal of Adolescent Health, 22, 56-65.

Thornberry, T. P., Smith, C. A., \& Howard, G. J. (1997). Risk factors for teenage fatherhood. Journal of Marriage and the Family, 59(3), 505-522.

Yogman, M. W., Kindlon, D., \& Earls, F. (1996). “The role of fathers”: Reply. Journal of the American Academy of Child and Adolescent Psychiatry, 35(6), 700.

Weatherley, R. A. (1991). Comprehensive services for pregnant and parenting adolescents: Historical and political considerations [Special Issue: Services to teenage parents]. Evaluation and Program Planning, 14(1-2), 17-25. 